\title{
Validation of the French-Canadian version of the Expanded Prostate Cancer Index Composite (EPIC) in a French-Canadian population
}

Éric Vigneault ${ }^{1,2}$; Josée Savard ${ }^{1,3,4}$; Marie-Hélène Savard ${ }^{1,3}$; Hans Ivers ${ }^{1,3,4}$; Philippe Després $^{1,2,3,5}$; William Foster ${ }^{2}$; André-Guy Martin ${ }^{1,2}$; Vincent Fradet ${ }^{1,3,6}$

${ }^{1} \mathrm{CHU}$ de Québec, Université Laval Research Centre; ${ }^{2}$ Department of Radiation Oncology, CHU de Québec, Université Laval; ${ }^{3}$ Université Laval Cancer Research Centre; ${ }^{4}$ School of Psychology, Université Laval;

${ }^{5}$ Département de physique, de génie physique et d’optique, Université Laval; ${ }^{6}$ Department of Surgery, Université Laval; Quebec City, QC, Canada

Acknowledgements: We sincerely thank the patients for their participation in this study and Fred Sengmueller for revising the manuscript. We also thank Josée Allard, Isabelle Desrosiers, Caroline Fortin, William Gilbert, Hélène Hovington, Jessica Julien, Véronique Massicotte, Karolane Renauld, Véronique Roy, and Eugénie Simard for their contribution to the project.

Support/Financial Disclosures: This study was supported by an ACURA Uro-Oncologic Radiation Awards held by the first, second, and last authors.

Cite as: Can Urol Assoc J 2017; Epub ahead of print. http://dx.doi.org/10.5489/cuaj.4428

Published online November 1, 2017

\section{Abstract}

Introduction: This study aims to empirically validate the French-Canadian version of the Expanded Prostate Cancer Index Composite (EPIC), a measure of health-related quality of life for prostate cancer patients.

Methods: 251 participants completed a battery of self-report scales including the FrenchCanadian version of the EPIC, after having received radiation therapy or radical prostatectomy for prostate cancer.

Results: The internal consistency for the urinary incontinence, bowel, and sexual domains of the EPIC-26 was high (Cronbach's alpha coefficients from .80 to .92), while coefficients for the urinary irritation/obstruction (.59) and hormonal (.67) domains were lower. Item-total correlations ( $r s=.15$ to .85 ), and temporal stability ( $r s=.72$ to .93 ) generally supported the reliability of the instrument. The 5-factor structure of the EPIC-26 was confirmed for the most part. The construct validity of the instrument was also supported by high correlations obtained between each domain and measures assessing similar constructs ( $r s=-.56$ to .83). The EPIC also showed an excellent sensitivity to change with significant differences obtained on EPIC scores (all $p s<.05$ ) between pre- and post-prostate cancer treatment.

Conclusions: The psychometric qualities of the French-Canadian version of the EPIC are well supported, thus providing a valid tool to assess HRQOL in prostate cancer patients. 


\section{Introduction}

Prostate cancer is the most common cancer in North-American men. Approximately 180,890 cases will be diagnosed in 2016 in the USA. ${ }^{1}$ While some low-risk patients may be put on active surveillance programs, intermediate and high-risk prostate cancer usually requires treatment. Several options are available including radical prostatectomy, external beam radiation therapy and brachytherapy. While these treatment modalities have demonstrated their efficacy in treating prostate cancer, they are associated with some toxicity and long-term side-effects. ${ }^{2-4}$ Healthrelated quality of life (HRQOL) is an important parameter of treatment success and is now typically assessed in standard practices. ${ }^{5}$ It is also an important aspect to take into account when helping patients choose the most appropriate treatment option for their condition.

The Expanded Prostate Cancer Index Composite (EPIC), a self-report scale, was designed to measure HRQOL specifically among prostate cancer patients. ${ }^{6}$ The original English version is composed of 50 questions encompassing 4 domains (urinary, bowel, sexual, and hormonal). Function and bother are assessed with different items within each domain, and the urinary domain comprises two additional subscales: incontinence and irritation/obstruction. Domain-specific standardized scores range from 0 to 100, a higher score indicating a better perceived quality of life. High Cronbach's alpha coefficients ( $>.82$, a measure of internal consistency ranging from 0 to 1 ) and a very good test-retest reliability ( $r s>.80$ ) were found for all domains. The EPIC-50 also has demonstrated an adequate validity with instruments assessing similar content (convergent validity) and other instruments assessing physical symptoms that are not specific to prostate cancer (divergent validity). ${ }^{6}$ In addition, this evaluation tool has been found to be sensitive to change following prostate cancer treatment. ${ }^{7}$

A shorter 26-item version (EPIC-26) was developed to facilitate its use in a wide range of prostate cancer research and practice settings. The EPIC-26 is highly correlated with the full questionnaire ( $r \mathrm{~s}>.96$ for all domains). ${ }^{8}$ This abbreviated version comprises five domains rather than four: urinary incontinence, urinary irritation/obstruction, bowel, sexual, and vitality/hormonal. The EPIC-26 has shown high internal consistency (Cronbach's alpha=.70-.90) and test-retest reliability at 2-4 week intervals ( $r s=.69-.90$ for all 5 domains). Moderate to strong correlations were found between the sexual domain and the Sexual Health Inventory for Men and between the urinary domain and the Incontinence Symptom Index, thus supporting its convergent validity. ${ }^{9}$

Two systematic comparisons of available instruments supported the use of the EPIC for the assessment of prostate-cancer specific HRQOL. ${ }^{10,11}$ The EPIC has been validated in several languages (i.e., Norwegian, Spanish, Korean). ${ }^{12-14}$ A French version is available, but only the EPIC-50 was validated. ${ }^{15}$ Validating the short version is critical as this is the version most likely to be used in clinical contexts, as well as in randomized controlled trials. Also, given significant cross-cultural language differences between French people and French-Canadians, a FrenchCanadian validation of the EPIC was needed.

This study aimed to translate the EPIC into French, as well as to assess its psychometric properties among a clinical sample of French-Canadian prostate cancer patients. For purposes of 
parsimony, only the results pertaining to the EPIC-26 will be described as this is the format typically used in clinics, although validation analyses were performed for both formats (see Appendix for results pertaining to the EPIC-50).

\section{Methods}

\section{Language equivalence}

An initial in-house French version of the EPIC-26 was developed and used for many years in the radiation oncology department of CHU de Québec-Université Laval. To verify its quality, this version was sent to two professional English-French translators whose native language is French, following recommendations by Haccoun. ${ }^{16}$ Both translators assessed whether each of the items and response choices was correctly translated, and suggested an alternative wording when needed. Based on the translators' suggestions, these items were then reformulated by our research team (JS, EV, MHS). Translators were also asked to translate into French the 24 additional items of the EPIC-50. One of the two proposed French versions of each item or a version combining the two proposed formulations was retained.

Next, English and French versions of all items were sent to two different English-French translators whose native language is French (Canadian) to assess to what extent each item was correctly translated. The same strategy as described above was used to reach a single translation.

\section{Pilot study}

This preliminary French version of the EPIC-50 was tested among a sample of 10 prostate cancer patients. Participants were recruited during a medical appointment at the radiation oncology department of CHU de Québec-Université Laval by a research assistant. Patients were eligible when they were scheduled to receive or were currently receiving radiation therapy. Patients provided informed consent prior to their participation. They were asked to complete the EPIC while verbalizing out loud any comment about the clarity of the items or the response choices to the research assistant who was noted down their comments.

Only the French translation of "dribbling/dripping", used in one question and two response choices, was noted as lacking clarity by three participants, and was therefore slightly modified to yield the final version.

\section{Empirical validation}

\section{Participants}

French-Canadian prostate cancer patients were recruited at the radiation oncology and the urooncology departments of CHU de Québec-Université Laval from March 2014 to January 2015. Patients were solicited in person by a research nurse during a medical appointment at the clinic.

Inclusion criteria: (a) having received radiation treatments (external beam radiation therapy or brachytherapy) and/or a radical prostatectomy (RP) for prostate cancer in the past OR to be scheduled to receive radiation treatments (for sensitivity to change analyses only); (b) having a life expectancy > 1 year; and (c) to be readily able to read and understand French. This study was 
approved by the research ethics board of the CHU de Québec-Université Laval and patients provided informed consent prior to their participation.

\section{Measures}

All of the following measures were validated in French or official French versions were used.

International Prostate Symptom Score (IPSS). ${ }^{17,18}$ This 7-item questionnaire assesses irritative/obstructive urinary prostate symptoms. Items are scored on a scale ranging from 0 (“never”) to 5 (“almost always”). The total score ranges from 0 to 35 , a lower score indicating a better functioning.

Sexual Health Inventory for Men (SHIM). ${ }^{19}$ This questionnaire is an abbreviated 5-item version of the 15-item International Index of Erectile Function. ${ }^{20}$ It assesses erectile function and intercourse satisfaction. Scores range from 1 to 5 (item 1) or from 0 to 5 (items 2-5), with a total score ranging from 1 to 25. Higher values indicate a better sexual functioning.

European Organization for Research and Treatment of Cancer Quality of Life Questionnaire - Prostate Cancer-Specific Module (PR25). ${ }^{21}$ This 25-item questionnaire assesses HRQOL of prostate cancer patients over the past week (20 items) or the last 4 weeks (5 items). It comprises two functional scales (sexual activity and sexual functioning) and four symptom scales (urinary, bowel, hormonal treatment-related, incontinence aid). Items are scored on a Likert scale ranging from 1 ("not at all") to 4 ("very much"). Standardized scores range from 0 to 100 , a higher score indicating a better (functioning scales) or worse (symptom scales) quality of life.

\section{Procedure}

Participants taking part in the sensitivity to change study $(n=51)$ were asked by the research nurse to complete the EPIC-50 along with the other self-report scales at their initial consultation, prior to receiving radiation therapy, and to fill out the EPIC-50 a second time during a follow-up visit at the hospital, approximately 3 months after their treatment ended ( $M=98.2$ days; range $=59-163$ days).

The remaining participants $(n=200)$ completed the same battery of questionnaires at a follow-up appointment with their radiation oncologist or surgical urologist. A subgroup of these participants $(n=75)$ was randomly selected to fill out the EPIC-50 on a second occasion, 2 weeks later, for test-retest reliability analyses. The randomization sequence was prepared by a biostatistician and the allocation sequence was concealed in opaque, sealed envelopes, numbered in advance and opened sequentially. All research personnel were blind to the group allocation sequence. These randomly selected participants were given a copy of the EPIC-50 to take home and were instructed to complete it 2 weeks later and to return it by mail. Participants who did not return the questionnaire within 4 weeks were contacted by phone to remind them to complete and return it as soon as possible. A total of 68 participants returned the second questionnaire (median interval=21 days; range=7-41). 


\section{Statistical analyses}

All data were double-entered and missing data, outliers, and distributions were examined using standard procedures. ${ }^{22}$ No data imputation was performed and the alpha level was set at 5\%, twotailed. All analyses were conducted on both the full (EPIC-50) and the abbreviated (EPIC-26) scales using the SAS 9.3 software (2012, SAS Institute, Cary, NC, USA). EPIC-26 items were extracted from the 50 -item version as the items are the same. All results pertaining to the EPIC26, the version most likely to be used in clinical and research settings, are discussed in this article. Detailed results about the 50-item version can be found in the Appendix.

Reliability (internal consistency). The Cronbach alpha coefficient ${ }^{23}$ and item-total correlations were calculated for each EPIC domain using the full sample $(N=251)$. An alpha $>$ .80 and item-total correlations $>.30$ (moderate association $^{24}$ ) were used to indicate an acceptable internal consistency.

Test-retest reliability. Correlations were computed between EPIC scores obtained on each domain on two different occasions, separated by a 2-4 week interval. In addition, scores between the two administrations were compared using linear mixed models with one time factor (two levels). A large correlation between the two time points $\left(r>.50^{24}\right)$ and a non-significant $F$ test were the criteria used to support the EPIC temporal stability.

Construct validity (factor analysis). A confirmatory factor analysis using SAS PROC CALIS (structural equation modeling) was performed using the full sample $(N=251)$ in order to verify the reproducibility of the factor structure of the EPIC-26 (5 domains: urinary incontinence, urinary irritation/obstruction, bowel, sexual, and vitality/hormonal symptoms) and the EPIC-50 (4 domains: urinary, bowel, sexual, hormonal). Various indices were examined to ensure an acceptable goodness of fit with the factorial structure of the original English version.

Convergent and divergent validity. The convergent validity of each domain of the EPIC26 and EPIC-50 was evaluated by assessing its relationship with other measures of similar constructs: the IPSS for the urinary irritation/obstruction domain; the SHIM for the sexual domain; and the PR25 for the other three domains (urinary incontinence, bowel, and vitality/hormonal domains). The divergent validity was evaluated by examining associations with different constructs, using the same questionnaires as for the convergent validity assessment (cross-correlations; e.g., between the urinary incontinence domain of the EPIC and the SHIM which assesses sexual symptoms). Lower (or non-significant) Pearson correlations were expected as compared to convergent validity correlations.

Sensitivity to change. In order to assess the capacity of the EPIC to detect changes in symptoms following treatment, 51 patients were asked to complete the EPIC-50 prior to the initiation of radiotherapy and approximately 12 weeks following its termination. A linear mixed model analysis with repeated measures was conducted. The presence of significant differences between pre- and post-treatment assessments on each domain was expected. 


\section{Results}

\section{Participant characteristics}

A total of 251 prostate cancer patients were included. Of these, 51 participated in the sensitivity to change study, whereas 68 other men were randomly selected to participate in the study's testretest component.

Participants were between 46 and 89 years old $(M=68.7 ; S D=7.39)$. The majority were married/cohabiting (73.3\%) and retired from work (73.4\%). Forty percent of the sample had a personal annual income lower than $\$ 40,000$ CAN. Thirty-four percent $(n=86)$ of the total sample were treated with a combination of external beam radiotherapy and brachytherapy, $18 \%(n=46)$ with radiotherapy only, $16 \%(n=39)$ with brachytherapy only, while $21 \%(n=53)$ received a combination of RP and radiotherapy, and the remaining 11\% ( $n=27)$ had RP only. Similar proportions were found in the sample that participated in the test-retest substudy (36.0\% radiotherapy and brachytherapy; $13.3 \%$ radiotherapy only; $18.7 \%$ brachytherapy only; $18.7 \% \mathrm{RP}$ and radiotherapy; $13.3 \% \mathrm{RP}$ only). Among the total sample, $46.2 \%$ of participants $(\mathrm{n}=116)$ received hormone therapy (current use: $21.6 \%$ ). The median time since the initial cancer diagnosis was 34.6 months (range $=0.3$ to 320 months), and median time since the end of treatment was 35 months (range $=1$ to 233 months). Fifteen percent of the participants had had a prostate cancer recurrence before their study participation.

\section{Descriptive statistics}

Table 1 shows the descriptive statistics for each EPIC-26 domain. Mean scores ranged from 34.9 for the sexual function subscale to 92.4 for the bowel bother subscale, a higher score indicating a better functioning (see Table S1 for EPIC-50).

Reliability

Internal consistency. Table 1 also shows the Cronbach's alpha coefficients and item-total correlations obtained for each EPIC-26 domain (see Table S1 for EPIC-50). Coefficients obtained for the urinary incontinence, bowel, and sexual domains were high (from .84 to .92), while coefficients for the urinary irritation/obstruction (.59) and hormonal (.67) domains were lower. Items with the lowest item-total correlations were \#4c (hematuria; $r=.15$ ), \#6d (bloody stools; $r=.33$ ), \#12 (overall sexuality problem; $r=.31$ ), and \#13b (breast problems; $r=.11$ ).

Test-retest reliability. Large correlations were found between EPIC-26 mean scores obtained at the first assessment and 2-4 weeks later ( $r$ s ranging from .72 to .93) and no significant differences were found between the two administrations (all ps>.40; see Table 2 [and Table S2 for EPIC-50]).

\section{Construct validity}

Confirmatory factor analysis. A structural equation model with five latent variables (the five postulated factors of the original EPIC-26) and all 10 pairwise covariances between the five latent variables was estimated using the maximum likelihood method. An adequate fit with the empirical variance-covariance matrix of the 26 items was found, $X^{2}(N=251, d f=284)=560.03$, 
$\mathrm{p}<.001$, chi-square/df ratio=1.97, Standardized Root Mean Square Residuals (SRMSR) $=0.069$, Bentler Comparative Fit Index $(\mathrm{CFI})=0.919$, Normed Fit Index $=0.850$. Correlations obtained between latent factors ranged from .23 to .48 , with stronger correlations found between urinary incontinence and urinary irritation/obstruction scales, $r=.69$, and between bowel and hormonal scales, $r=.51$ (see standardized loadings in Table 3). Only two items were weakly associated $(\mathrm{B}<.30)$ with their postulated factor: item \#4c of the urinary irritation/obstruction scale (hematuria), $\mathrm{B}=0.201$, and item \#13b of the hormonal scale (breast problems), $\mathrm{B}=0.155$.

For the EPIC-50, a structural equation model with four latent variables and all 6 pairwise covariances between the latent variables was estimated. A poor fit with the empirical variancecovariance matrix of the 50 items was found, $\mathrm{X}^{2}(N=251, d f=1165)=4538.6, \mathrm{p}<.001$, chi-square/df ratio=3.90, Standardized Root Mean Square Residuals (SRMSR)=0.099, Bentler Comparative Fit Index $(\mathrm{CFI})=0.582$, Normed Fit Index $=0.512$. Seven items were weakly associated with their postulated factor: items \#2 ( $\mathrm{B}=.26)$, \#3 $(\mathrm{B}=.27)$, and \#8 $(\mathrm{B}=.28)$ of the urinary scales, item \#18 (.23) of the bowel scale, and items \#41 $(B=.24)$, \#44 $(B=.25)$ and \#46 $(B=.20)$ of the hormonal scale (see Table S3).

Convergent and divergent validity. As seen in Table 4 (Table S4 for EPIC-50), the correlations obtained between scores on EPIC-26 domains and those found on questionnaires measuring similar constructs were high ( $r$ s between .56 and .83 , all $p s<.05$ ). In contrast, the correlations obtained with questionnaires/subscales measuring different constructs were consistently weaker. For instance, the correlation obtained between the EPIC-26 sexual domain and the urinary, bowel, and hormonal subscales of the IPSS or the PR25 were lower ( $r$ s between -.19 and .36) than with the SHIM or the PR25 sexual subscales ( $r$ s between .63 and .83). Sensitivity to Change

As shown in Table 2 (Table S2 for EPIC-50), scores obtained on each EPIC-26 domain significantly decreased after radiotherapy treatment (all $p s<.01$ ), suggesting a worsening of HRQOL.

\section{Discussion}

The objective of this study was to validate empirically the French-Canadian version of the EPIC. Overall, the psychometric properties found in this study are similar to those of the original English version. ${ }^{6,8}$ Hence, results suggest that this French version is a reliable and valid instrument for assessing prostate cancer-related symptoms.

Test-retest correlations at an interval of 2 to 4 weeks were high across all domains, suggesting an excellent reliability. Cronbach's alpha coefficients for the urinary incontinence, bowel, and sexual domains were high, whereas they were lower for the urinary irritation/obstruction and hormonal domains, suggesting weaker internal consistency for these two scales. These lower coefficients seem to be due to two more problematic items, that is hematuria (urinary - irritation/obstruction) and breast problems (hormonal) which seem to assess different constructs than their respective domain. They were also not frequently reported in our sample. 
The 5-factor structure of the original EPIC-26 was in large part confirmed, ${ }^{8}$ with the same two items being weakly correlated with their postulated factor (hematuria and breast problems). The 4-factor structure of the EPIC-50 was less sound with poor fit indices and seven items that loaded less strongly with their respective factor. These include three items in the urinary domain (two hematuria items and one dysuria item), one in the intestinal domain (bowel movements frequency) and three in the hormonal domain, the latest being the least strongly loaded with their postulated factor. It is interesting to note that these findings are consistent with those of Anota et al. ${ }^{15}$ who, although they did not perform a confirmatory factor analysis, found that the hormonal domain of their French version presented a poor construct validity overall. Similarly, the authors of the Korean version of the EPIC-50 also observed some disparity in the factorial structure of this subscale. ${ }^{14}$ Together, these findings question the composition of the hormonal subscale.

The convergent validity of the EPIC was also well supported. Indeed, for each of the five domains of the EPIC-26, the highest correlations (all>.55) were found with instruments measuring similar constructs, whereas lower correlations $($ all $<.55)$ were consistently found with instruments measuring different constructs. Clearly, the French version of the EPIC accurately measures distinct domains that are relevant to prostate cancer-related quality of life.

Moreover, the French-Canadian version of the EPIC proved to be sensitive to clinical changes associated with prostate cancer treatment. All domain scores significantly decreased following radiotherapy, which is consistent with the bulk of evidence published on this instrument showing its good sensitivity. ${ }^{25}$ Also, the magnitude of the differences observed between pre- and post-treatment data were all above the thresholds for a clinically relevant change (Minimally Important Difference) proposed by Skolarus et al. for this instrument. ${ }^{26}$ This indicates that the French-Canadian version of the EPIC can be routinely used in clinics to track changes in patient-reported HRQOL associated with prostate cancer treatment.

The large sample size is a strength of this study, as well as the assessment of the translation quality and of various psychometric properties using rigorous and recognized procedures. On the other hand, no information was available on the number of patients who were approached in each clinic to document the participation rate, thus questioning the representativeness of the sample. Our sample, which received a variety of treatments alone and in combination, was fairly representative of the population of prostate cancer patients although recruiting a fully representative sample is a challenge in the context of this cancer. However, a representative sample is not critical to study the psychometric properties of an instrument, since correlations between items, and thus the internal structure of the instrument, are not expected to vary according to medical treatment options. Besides, the fact that EPIC scores significantly deteriorated after radiation therapy, a treatment usually associated with less sudden side effects than RP, provides convincing evidence of its sensitivity to clinical change.

Conclusion

The French-Canadian version of the EPIC, particularly its short-form (EPIC-26), is a reliable and valid measure of HRQOL in prostate cancer patients and can be used for both clinical and research purposes. The availability of this instrument in French will facilitate the 
assessment and management of patient-reported outcomes in francophone clinics and research settings. 


\section{References}

1. $\quad$ American Cancer Society. Cancer Facts \& Figures 2016.

2. Cooperberg MR, Broering JM, Carroll PR. Risk assessment for prostate cancer metastasis and mortality at the time of diagnosis. J Natl Cancer Inst 2009;101:878-87.

3. Klein EA, Ciezki J, Kupelian PA, et al. Outcomes for intermediate risk prostate cancer: Are there advantages for surgery, external radiation, or brachytherapy? Urol Oncol 2009;27:67-71.

4. Donovan JL, Hamdy FC, Lane JA, et al. Patient-reported outcomes after monitoring, surgery, or radiotherapy for prostate cancer. N Engl J Med 2016;375:1425-37.

5. Bottomley A, Therasse P. Quality of life in patients undergoing systemic therapy for advanced breast cancer. Lancet Oncol 2002;3:620-8.

6. Wei JT, Dunn RL, Litwin MS, et al. Development and validation of the Expanded Prostate Cancer Index Composite (EPIC) for comprehensive assessment of health-related quality of life in men with prostate cancer. Urology 2000;56:899-905.

7. Wei JT, Dunn RL, Sandler HM, et al. Comprehensive comparison of health-related quality of life after contemporary therapies for localized prostate cancer. J Clin Oncol 2002;20:557-66.

8. Szymanski KM, Wei JT, Dunn RL, et al. Development and validation of an abbreviated version of the Expanded Prostate Cancer Index Composite instrument (EPIC-26) for measuring health-related quality of life among prostate cancer survivors. Urology 2010;76:1245-50.

9. Hedgepeth RC, Labo J, Zhang L, et al. Expanded Prostate Cancer Index Composite versus Incontinence Symptom Index and Sexual Health Inventory for Men to measure functional outcomes after prostatectomy. J Urol 2009;182:221-7.

10. Rnic K, Linden W, Tudor I, et al. Measuring symptoms in localized prostate cancer: A systematic review of assessment instruments. Prostate Cancer Prostatic Dis 2013;16:111-22.

11. Schmidt S, Garin O, Pardo Y, et al. Assessing quality of life in patients with prostate cancer: a systematic and standardized comparison of available instruments. Qual Life Res 2014;23:2169-81.

12. Fossa SD, Storas AH, Steinsvik EA, et al. Psychometric testing of the Norwegian version of the Expanded Prostate Cancer Index Composite 26-item version (EPIC-26). Scand J Urol 2016:1-6.

13. Ferrer M, Garin O, Pera J, et al. [Evaluation of the quality of life of patients with localizad prostate cancer: validation of the Spanish version of the EPIC]. Med Clin (Barc) 2009;132:128-35.

14. Chung KJ, Kim JJ, Lim SH, et al. Development and validation of the korean version of Expanded Prostate Cancer Index Composite: Questionnaire assessing health-related quality of life after prostate cancer treatment. Korean J Urol 2010;51:601-12. 
15. Anota A, Mariet AS, Maingon P, et al. Cross-cultural adaptation and validation of the French version of the Expanded Prostate cancer Index Composite questionnaire for health-related quality of life in prostate cancer patients. Health Qual Life Outcomes 2016;14:168.

16. Haccoun RR. Une nouvelle technique de vérification de l'équivalence de mesures psychologiques traduites. Revue Québécoise de Psychologie 1987;8:30-9.

17. Cockett ATK, Aso Y, Denis L, et al. Recommendations of the Internaltional Consensus Committee concerning: 1. Prostate Symptom Score (I-PSS) and Quality of Life Assessment. In: Cockett ATK, Aso Y, Chatelain C, al. e, eds. Proceedings of the International Consultation on Bening Prostatic Hyperplasia (BPH). Paris, France1992:279-88.

18. Gregoire JP, Moisan J, Labrecque M, et al. [Validation of a French adaptation of the International Prostatic Symptom Score]. Prog Urol 1996;6:240-9.

19. Rosen RC, Cappelleri JC, Smith MD, et al. Development and evaluation of an abridged, 5-item version of the International Index of Erectile Function (IIEF-5) as a diagnostic tool for erectile dysfunction. Int J Impot Res 1999;11:319-26.

20. Rosen RC, Riley A, Wagner G, et al. The International Index of Erectile Function (IIEF): A multidimensional scale for assessment of erectile dysfunction. Urology 1997;49:82230.

21. van Andel G, Bottomley A, Fossa SD, et al. An international field study of the EORTC QLQ-PR25: A questionnaire for assessing the health-related quality of life of patients with prostate cancer. Eur J Cancer 2008;44:2418-124.

22. Tabachnick BG, Fidell LS. Using multivariate statistics 6th ed. New-York: Allyn \& Bacon; 2012.

23. Cronbach LJ. Essentials of psychological testing. 4th ed. New-York: Harper \& Row Publishers; 1984.

24. Cohen J. Statistical power analysis for the behavioral sciences. 2nd ed. Hilesdale, NJ: Lawrence Erlbaum Associates; 1988.

25. Lee TK, Breau RH, Mallick R, et al. A systematic review of expanded prostate cancer index composite (EPIC) quality of life after surgery or radiation treatment. Can J Urol 2015;22:7599-606.

26. Skolarus TA, Dunn RL, Sanda MG, et al. Minimally important difference for the Expanded Prostate Cancer Index Composite Short Form. Urology 2015;85:101-5. 


\section{Figures and Tables}

\begin{tabular}{|l|l|c|c|c|c|c|c|}
\hline $\begin{array}{l}\text { Table 1. Descriptive statistics obtained on the EPIC-26 and results of internal consistency } \\
\text { analyses (n=251) }\end{array}$ & $\mathrm{n}$ & $\begin{array}{c}\text { Mean score } \\
\text { (T1) }\end{array}$ & SD & Median & Range & $\begin{array}{c}\text { Cronbach's } \\
\text { alpha }\end{array}$ & Range $r_{\text {(tot) }}$ \\
\hline Domains & 241 & 86.39 & 21.84 & 100.00 & $0-100$ & 0.889 & $0.74-0.84$ \\
\hline $\begin{array}{l}\text { Urinary } \\
\text { incontinence }\end{array}$ & 241 & 87.42 & 13.97 & 93.75 & $12.5-100$ & 0.594 & $0.15-0.51$ \\
\hline $\begin{array}{l}\text { Urinary } \\
\text { irritation/ } \\
\text { obstruction }\end{array}$ & 250 & 78.60 & 27.02 & 87.50 & $0-100$ & NA & NA \\
\hline $\begin{array}{l}\text { Urinary overall } \\
\text { problem }\end{array}$ & 245 & 92.38 & 12.86 & 100.00 & $0-100$ & 0.837 & $0.33-0.77$ \\
\hline $\begin{array}{l}\text { Bowel } \\
\text { Sexual }\end{array}$ & 246 & 34.87 & 28.77 & 25.00 & $20.83-100$ & 0.887 & $0.31-0.85$ \\
\hline Hormonal & 246 & 89.92 & 14.10 & 95.00 & $0-100$ & 0.670 & $0.11-0.74$ \\
\hline
\end{tabular}

EPIC: Expanded Prostate Cancer Index Composite; $r_{\text {(tot) }}$ : item-total correlations; NA: not applicable (only one item). 


\begin{tabular}{|c|c|c|c|c|}
\hline Domains & $\begin{array}{c}\text { Mean score } \\
\text { (T1) }\end{array}$ & $\begin{array}{c}\text { Mean score } \\
\text { (T2) }\end{array}$ & F-test & r (T1 vs. T2) \\
\hline \multicolumn{5}{|l|}{ Test/re-test analyses $(n=68)$} \\
\hline Urinary incontinence & 84.42 & 84.63 & $0.04, p=0.83$ & 0.93 \\
\hline Urinary irritation/obstruction & 87.31 & 86.47 & $0.69, p=0.41$ & 0.78 \\
\hline Urinary overall problem & 77.76 & 78.55 & $0.11, p=0.74$ & 0.72 \\
\hline Bowel & 92.02 & 92.61 & $0.50, p=0.48$ & 0.86 \\
\hline Sexual & 30.76 & 30.46 & $0.06, p=0.81$ & 0.92 \\
\hline Hormonal & 89.63 & 89.70 & $0.00, p=0.95$ & 0.75 \\
\hline \multicolumn{5}{|c|}{ Sensitivity to change analyses $(n=51)$} \\
\hline Urinary incontinence & 95.10 & 88.19 & $6.77, p=0.010$ & 0.46 \\
\hline Urinary irritation/obstruction & 87.93 & 81.59 & $8.21, \mathrm{p}=0.006$ & 0.45 \\
\hline Urinary overall problem & 81.86 & 69.61 & 9.46, $\mathrm{p}=0.003$ & 0.46 \\
\hline Bowel & 94.00 & 86.54 & $11.48, \mathrm{p}=0.001$ & 0.33 \\
\hline Sexual & 50.58 & 29.92 & 25.59, $\mathrm{p}<0.001$ & 0.47 \\
\hline Hormonal & 91.20 & 82.29 & 19.70, $\mathrm{p}<0.001$ & 0.52 \\
\hline
\end{tabular}




\begin{tabular}{|c|c|c|c|}
\hline Item & $\begin{array}{c}\text { \#item } \\
\text { EPIC-50 }\end{array}$ & $\begin{array}{c}\text { \#item } \\
\text { EPIC- } \\
26\end{array}$ & $\begin{array}{c}\text { Standard } \\
\text { loading }\end{array}$ \\
\hline \multicolumn{4}{|l|}{ URINARY - incontinence } \\
\hline Leaking $>1$ time per day & 1 & 1 & 0.855 \\
\hline Frequent dribbling & 4 & 2 & 0.825 \\
\hline Any pad use & 5 & 3 & 0.764 \\
\hline Leaking problem & 6 & $4 a$ & 0.938 \\
\hline \multicolumn{2}{|c|}{ URINARY - irritation/obstruction } & 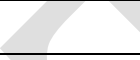 & \\
\hline Dysuria & 7 & $4 \mathrm{~b}$ & 0.491 \\
\hline Hematuria & 8 & $4 \mathrm{c}$ & 0.201 \\
\hline Weak stream & 9 & $4 d$ & 0.559 \\
\hline Frequency & 11 & $4 e$ & 0.717 \\
\hline OVERALL urinary problem & 12 & 5 & 0.928 \\
\hline \multicolumn{4}{|l|}{ BOWEL } \\
\hline Urgency & 20 & $6 a$ & 0.864 \\
\hline Frequency & 21 & $6 \mathrm{~b}$ & 0.760 \\
\hline Fecal incontinence & 23 & 6c & 0.697 \\
\hline Bloody stools & 24 & $6 \mathrm{~d}$ & 0.357 \\
\hline Rectal pain & 25 & $6 e$ & 0.558 \\
\hline Overall bowel problem & 26 & 7 & 0.810 \\
\hline \multicolumn{4}{|l|}{ SEXUAL } \\
\hline Poor erections & 28 & $8 a$ & 0.912 \\
\hline Difficulty with orgasm & 29 & $8 \mathrm{~b}$ & 0.847 \\
\hline Erections not firm & 30 & 9 & 0.838 \\
\hline Erections not reliable & 31 & 10 & 0.908 \\
\hline Poor sexual function & 35 & 11 & 0.818 \\
\hline Overall sexuality problem & 39 & 12 & 0.326 \\
\hline \multicolumn{4}{|l|}{ HORMONAL } \\
\hline Hot flashes & 45 & $13 a$ & 0.479 \\
\hline Breast problems & 46 & $13 \mathrm{~b}$ & 0.155 \\
\hline Depression & 48 & $13 c$ & 0.685 \\
\hline Lack of energy & 49 & $13 \mathrm{~d}$ & 0.938 \\
\hline Weight change & 50 & $13 \mathrm{e}$ & 0.448 \\
\hline
\end{tabular}

All loadings were significant at $\mathrm{p}<0.05$. 


\begin{tabular}{|c|c|c|c|c|c|c|c|c|c|}
\hline Domain & $\begin{array}{c}\text { IPSS } \\
\text { total } \\
(n=206) \\
\end{array}$ & $\begin{array}{c}\text { IPSS } \\
\text { QoL } \\
(n=203)\end{array}$ & $\begin{array}{c}\text { SHIM } \\
(n=203)\end{array}$ & $\begin{array}{c}\text { PR25- } \\
\text { SAC }^{f} \\
(n=199)\end{array}$ & $\begin{array}{c}\text { PR25- } \\
\text { SFU }^{f} \\
(n=117)\end{array}$ & $\begin{array}{c}\text { PR25- } \\
\text { URI }^{\text {s }} \\
(n=204)\end{array}$ & $\begin{array}{c}\text { PR25- } \\
\text { BOW }^{\text {s }} \\
(\mathbf{n}=\mathbf{2 0 0})\end{array}$ & $\begin{array}{c}\text { PR25- }^{-} \\
\text {HTR }^{\text {s }} \\
(n=204)\end{array}$ & $\begin{array}{c}\text { PR25- } \\
\text { AID }^{s} \\
(n=40)\end{array}$ \\
\hline $\begin{array}{l}\text { Urinary } \\
\text { incontinence }\end{array}$ & -0.44 & -0.39 & 0.27 & 0.16 & 0.27 & -0.65 & -0.34 & -0.28 & -0.70 \\
\hline $\begin{array}{l}\text { Urinary } \\
\text { irritation/ } \\
\text { obstruction }\end{array}$ & -0.76 & -0.56 & 0.21 & 0.17 & 0.25 & -0.71 & -0.38 & -0.31 & $\begin{array}{c}-0.31 \\
\mathrm{NS}\end{array}$ \\
\hline $\begin{array}{l}\text { Urinary overall } \\
\text { problem }\end{array}$ & -0.68 & -0.61 & 0.24 & 0.19 & 0.30 & -0.78 & -0.26 & -0.25 & -0.71 \\
\hline Bowel & -0.52 & -0.35 & 0.18 & 0.14 & 0.26 & -0.49 & -0.65 & -0.37 & $\begin{array}{c}-0.30 \\
\text { NS }\end{array}$ \\
\hline Sexual & -0.21 & -0.34 & 0.83 & 0.63 & 0.72 & -0.34 & -0.25 & -0.36 & $\begin{array}{c}-0.19 \\
\text { NS }\end{array}$ \\
\hline Hormonal & -0.47 & -0.34 & 0.24 & 0.19 & 0.35 & -0.45 & -0.56 & -0.62 & -0.37 \\
\hline
\end{tabular}

EPIC: Expanded Prostate Cancer Index Composite; IPSS: International Prostate Symptom Score; SHIM: Sexual Health Inventory for Men; PR25: Prostate cancer-specific module of the European Organization for Research and Treatment of Cancer Quality of Life Questionnaire; SAC : sexual activity (functioning scale); $\mathrm{SFU}^{\mathrm{f}:}$ sexual functioning (functioning scale); URI': urinary (symptom scale); BOW' : bowel (symptom scale); $\mathrm{HTR}^{\mathrm{s}:}$ hormonal treatment-related (symptom scale); AID ${ }^{\text {s: }}$ incontinence aid (symptom scale). Correlations used for convergent validity are in bold. All correlations are significant at $\mathrm{p}<0.05$ unless otherwise specified. 